\title{
The effects of probability ambiguity on preferences for uncertain two-outcome prospects
}

\author{
MARK F. STASSON, WILLIAM G. HAWKES, H. DAVID SMITH, and WALTER M. LAKEY \\ Virginia Commonwealth University, Richmond, Virginia
}

\begin{abstract}
Many studies have investigated judgment and decision making under uncertainty (i.e., when the possible outcomes of a given alternative occur with some known exact probability between 0 and 1). Fewer studies, however, have investigated judgment and decision making in which the probabilities associated with different outcomes are ambiguous (i.e., known to be in a certain interval such as .40-.60, rather than known to be a specific value such as .50). Judgment under both uncertainty and ambiguity was investigated by having subjects evaluate two-outcome prospects that varied in expected value, level of probability ambiguity, and the center of the ambiguous probability range. Results indicated a preference for unambiguous probabilities when the probability of obtaining the better outcome was .50 or greater and a preference for a small amount of ambiguity when the probability of obtaining the better outcome was moderately low (.30).
\end{abstract}

The process of making judgments and choices is one that occurs frequently in our lives. Whether we are evaluating two job offers, deciding whether or not to purchase a particular automobile, or judging the attractiveness of a new home or apartment, we are engaging in a process of determining our preferences in an uncertain environment-that is, we are rarely certain of the outcomes associated with the object or prospect. Research investigating judgment and decision in these types of contexts has often relied on the use of some version of (subjective) expected utility theory (see, e.g., von Neumann \& Morgenstern, 1947). The focus in these studies is typically on predicting a person's judgment or choice from the likelihood of and the person's evaluation of each outcome. This approach is generally referred to as the study of judgment under uncertainty because each choice alternative has more than one possible outcome and each outcome cannot be predicted with certainty but rather with some probability between 0 and 1 . Recently, some theorists have recognized that in many decision-making contexts, there exists a range or interval of probabilities for each possible outcome, something that has been called probability ambiguity (Becker \& Brownson, 1964; Curley \& Yates, 1985; Einhorn \& Hogarth, 1985). Although there have been several different definitions of "ambiguity" in the literature, in this paper we will use Becker and Brownson's (1964) definition of ambiguity as "any distribution of probabilities other than a point estimate" (p. 64).

The question of if and how probability ambiguity affects preferences is an important one today since deci-

\footnotetext{
This research was supported in part by the Grants-In-Aid Program for Faculty of Virginia Commonwealth University. Preliminary results from this study were presented at the 1991 Annual Meeting of the Society for Judgment and Decision Making, San Francisco. Correspondence concerning this article should be addressed to M. F. Stasson, Department of Psychology, Box 2018, Virginia Commonwealth University, Richmond, VA 23284-2018 (e-mail: mstasson@cabell.vcu.edu).
}

sion makers are more frequently provided with probability estimates that are ranges or intervals rather than simple point estimates. For example, the success of a medical treatment may be characterized as $40 \%-60 \%$ rather than as $50 \%$. Although recognizing variability in probability approximations and providing interval estimates seems to be an important and helpful aid to decision makers, research in a number of contexts has indicated that decisions and judgments may differ depending on whether one has point or interval probability information (see, e.g., Curley \& Yates, 1985; Gardenfors \& Sahlin, 1983). These studies typically compare responses to unambiguous prospects such as "receiving $\mathrm{X}$ with probability .5 or receiving Y with probability .5," with responses on ambiguous prospects such as "receiving $\mathrm{X}$ with some probability in the range .4 to .6 or receiving $\mathrm{Y}$ with some probability in the range .4 to .6 in which all probabilities in the range are equally likely."

One of the earliest theoretical approaches in the area of ambiguity and judgment was that of Ellsberg (1961), who noted that in many contexts people violate the predictions made by traditional probability theory (Savage, 1972). Ellsberg speculated that the decision maker would avoid ambiguity if given a choice between certain and ambiguous probabilities. This notion has been supported by research in a number of domains, and, in some cases, subjects have indicated a willingness to pay a premium for an unambiguous prospect in order to avoid an ambiguous prospect with an equivalent expected value (Becker \& Brownson, 1964). We should note that Ellsberg suggested (see Becker \& Brownson, 1964, p. 63) that people may prefer ambiguity when the probability of a favorable outcome is very low; however, no research has reported this ambiguity-seeking preference.

A study by Curley and Yates (1985) exemplifies research on ambiguity preference. These researchers presented subjects with pairs of prospects, each having two 
possible outcomes, that were equivalent except that the prospects differed in terms of probability ambiguity. Each prospect specified the possibilities of winning $\$ 5$ or winning nothing, but these researchers used prospects with probabilities that were unambiguous (the exact probabilities of the two outcomes were known), known to be in a range of \pm .10 (a range covering .21), or known to be in a range of \pm .20 (a range covering .41). Overall, people seemed to be ambiguity-avoidant, preferring the prospects with unambiguous probabilities. However, the level of ambiguity avoidance exhibited by subjects was dependent on the center of the probability range-subjects were ambiguity-avoidant only when the probability of winning $\$ 5$ (or the center of the probability range) was at least .50. Ambiguity level had no effect when the center of the probability range was less than .50 .

This study was designed to replicate and extend the results found by Curley and Yates (1985). We extended their study in three important ways. First, as many decision makers working under probability ambiguity have fairly precise estimates of the true probabilities, we decided to examine prospects with narrower probability intervals than those used by Curley and Yates. In this study, we used prospects with ambiguity intervals of \pm .08 (labeled extreme ambiguity), \pm .04 (labeled moderate ambiguity), or \pm .00 (exact probabilities, labeled unambiguous). Second, Curley and Yates used different probabilities (interval centers) of winning $\$ 5$, while holding the amount to be won constant. Thus, any differences between the interval centers could be attributed to differences among the probabilities of winning or to differences in the expected values of the prospects. In this study, we assessed the interactive effects of probability ambiguity with both expected value and probability of winning (interval center). We did this by varying the probability of winning $(.10, .30, .50, .70$, or .90$)$ while altering the amount to be won in such a way as to hold expected value constant. We examined expected value by constructing two sets of prospects with expected values of either $\$ 1.50$ or $\$ 2$. Third, for a given probability of winning (interval center), Curley and Yates presented all possible pairs of prospects differing in probability ambiguity and asked subjects which prospect they would rather play and the degree to which they preferred the chosen prospect to the unchosen prospect. Recognizing that peoples' judgments often exemplify " preference reversals" (Lichtenstein \& Slovic, 1971) when measured in different ways, we obtained independent ratings of each prospect on two bipolar scales to see if our results would be similar to those obtained previously. Also, this measurement procedure allowed us to see how preferences for prospects of equal expected value are influenced by the probability of getting the better outcome. We predict that people will prefer higher probabilities of winning, although they will win a smaller amount of money than when the probability of the better outcome is low (a "preference for certainty of winning" effect). Thus, we can informally compare the effect of this "certainty" effect with the effects of "ambiguity."

\section{METHOD}

\section{Subjects and Design}

The subjects were 39 students in an upper-level psychology class at Virginia Commonwealth University who received partial course credit and the chance to win some money in return for their participation. Six subjects who failed to rate one or more prospects were eliminated from the analysis, so the final sample consisted of 33 subjects. We constructed 30 prospects with two possible monetary outcomes: receiving no money with probability $1-p$ and receiving some amount of money with probability $p$. The prospects differed in terms of expected value $(\$ 1.50$ or $\$ 2$ ), the probability of winning some amount of money ( $p$, which could take on values of $.10, .30, .50, .70$, or .90$)$, and probability ambiguityprospects were unambiguous (exact probabilities known), "moderately" ambiguous (probabilities known to be in a range of \pm .04 ), or "extremely" ambiguous (probabilities known to be in a range of \pm .08 ).

\section{Procedure}

The subjects were tested in a large classroom in which they were seated with at least one empty desk between any 2 subjects. The subjects were given a booklet that listed the 30 prospects in a random order. The subjects were given response sheets on which they rated each prospect on two 21-point bipolar scales corresponding to the following questions: (1) Do you feel this is a good prospect or a bad prospect? and (2) How willing are you to play this prospect? The average of these ratings served as the preference measure, in which higher numbers reflected greater preference. ${ }^{1}$ After rating all 30 prospects, each subject played one of the prospects, received any winnings, was debriefed, and then dismissed.

\section{RESULTS}

The preference ratings were analyzed using a 2 (expected value) $\times 5$ (interval center) $\times 3$ (ambiguity level) analysis of variance. There was a significant main effect for interval center $[F(4,128)=54.89, p<.01]$. As one can see from the means plotted in Figure 1, the higher the probability of winning (interval center), the more the subjects preferred the prospect. The only other significant effect was an interaction between probability of winning (interval center) and level of ambiguity $[F(8,256)=$ $3.29, p<.01]$. At interval centers of .50 or more, the

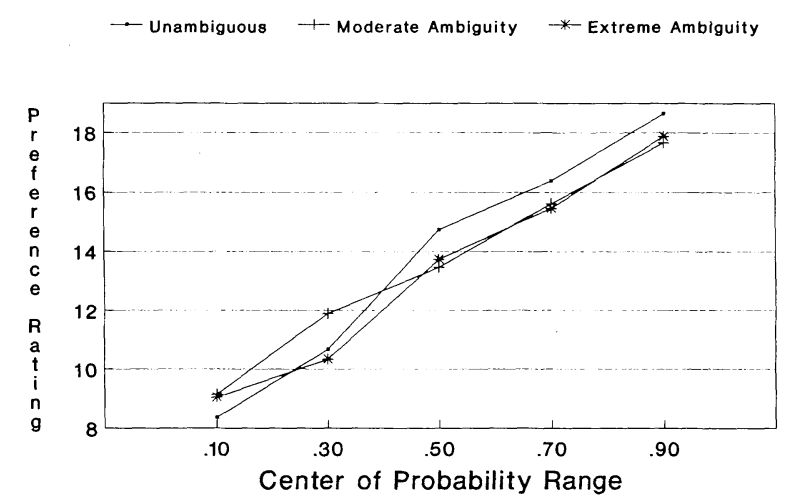

Figure 1. Mean preference rating as a function of a prospect's probability center and degree of ambiguity. Ratings were made on a 1-21 scale; higher values reflect greater preference. 
unambiguous prospect was preferred most, although this was significant only at interval centers of .50 and .90 . When the probability of winning was .10 or .30 , the prospect with moderate ambiguity was preferred most, although this was significant only at .30 . The expected value variable was not involved in any significant effects.

\section{DISCUSSION}

First, using relatively small ambiguity intervals spanning ranges of .09 and .17 (as compared with the .21 and .41 ranges used by Curley \& Yates, 1985), evidence of ambiguity avoidance was found when the center of the probability interval was .50 or more. Thus, at least when the probability of winning is high (.50 or more), even decision makers with fairly precise probability estimates still prefer prospects with exact probabilities to those that contain some ambiguity (as suggested by Ellsberg, 1961).

Second, after holding the expected values of prospects constant and manipulating both the center of the probability range and expected value, it was found that the center of the probability range most strongly affects preferences. This supports Curley and Yates' (1985) conclusions regarding the effects of interval center.

Third, when the probability center was .30 , the subjects in this study surprisingly preferred the moderately ambiguous prospects (probability intervals of .09) to unambiguous prospects and prospects with larger probability intervals (.17). This "ambiguity seeking" tendency was unexpected because prior research had found no such disposition (Curley \& Yates, 1985; Ellsberg, 1961), but it is interesting to see that when some ambiguity is preferred, subjects still do not prefer the most ambiguous prospect. It is unclear as to whether this ambiguity-seeking tendency is limited to the particular prospects used in this study, hence future work on this topic is needed to see if there are other cases in which people prefer ambiguity.

Fourth, there was a strong tendency for subjects to prefer prospects with higher probabilities of winning something, with each increase of .20 in the probability of winning translating into a roughly 2-point increase in preference scores. This "certainty of winning something" effect (albeit a smaller amount of money than would be won in prospects with lower probabilities of winning) seemed to be a bit stronger than the "ambiguity" effects found here as the differences in preference scores among the three ambiguity conditions was typically less than 1.5 scale points. This last point is made rather tentatively since probability levels were manipulated across a much wider range than were the ambiguity levels in this study.

The strongest aspect of this study seems to be that relatively small manipulations of probability ambiguity can produce effects on prospect preferences quite similar to the effects found in studies using very strong ambiguity manipulations (see, e.g., Curley \& Yates, 1985). It seems that people who are fairly confident about getting the desired outcome ( $50 \%$ chance or higher) prefer to avoid ambiguity because they do not want to consider "lower" chances of "winning." On the other hand, when it is "unlikely" that the desired outcome will occur (less than $50 \%$ chance) people do not avoid ambiguity and may even prefer some ambiguity so that the chances of "winning" may actually be "higher" than what an unambiguous prospect would offer. It may be that there is a place near the middle of the probability scale (e.g., $p=.50$ ) that serves as an inflection point at which ambiguity is disliked when $p$ is higher than this value and ambiguity is received more positively when $p$ is less than this value.

It is important to examine how people respond to ambiguity because it could have implications in decision-making situations in which people may select an action or alternative (e.g., choice of medical treatment, occupation, or investment) on the basis of how clearly the probability of a desired outcome is known. For example, there may be a tendency to choose established alternatives with precisely known outcome probabilities over alternatives that may potentially be better but are discarded because they have poorly defined outcome probabilities. The results here suggest the need for further research in this area to examine people's tolerance of ambiguity in a variety of decision settings.

\section{REFERENCES}

BeCKer, S. W., \& BRownson, F. O. (1964). What price ambiguity? Or the role of ambiguity in decision making. Journal of Political Economy, 72, 62-73.

Curley, S. P., \& Yates, J. F. (1985). The center and range of the probability interval as factors affecting ambiguity preferences. Organizational Behavior \& Human Decision Making Processes, 36, 273-287.

EINHORN, H. J., \& HogarTh, R. M. (1985). Ambiguity and uncertainty in probabilistic inference. Psychological Review, 92, 433-461.

ELLSBERG, D. (1961). Risk, ambiguity, and the savage axioms. Quarterly Journal of Economics, 75, 643-669.

Gardenfors, P., \& SAhLin, N.-E. (1983). Decision making with unreliable probabilities. British Journal of Mathematical \& Statistical Psychology, 36, 240-251.

Lichtenstein, S., \& SLovic, P. (1971). Reversals of preference between bids and choices in gambling decisions. Journal of Experimental Psychology, 89, 46-55.

SAVAGE, L. J. (1972). The foundations of statistics (2nd ed.). New York: Dover.

von Neumann, J., \& Morgenstern, O. (1947). Theory of games and economic behavior. Princeton, NJ: Princeton University Press.

\section{NOTE}

1. These two individual scales, which measure both the affective and behavioral components of preference, were highly correlated, and separate analyses of the individual scales yielded results similar to those for the composite variable.

(Manuscript received July 2, 1993.) 